We now present these results in tabular form.

\begin{tabular}{c|c|l}
\hline polynomial & $q=p^{n}$ & \multicolumn{1}{|c}{$G F(q)$ which are permuted } \\
\hline \multirow{3}{*}{$x^{8}+a x$} & $8 m+3$ & none \\
& $8 m+5$ & $G F(29)$ and no others \\
& $8 m+7$ & none \\
& $8 m+3$ & $G F(11)$ and possibly $G F\left(11^{n}\right)$ for odd $n$ \\
$x^{8}+a x^{3}$ & $8 m+5$ & none \\
& $8 m+7$ & none \\
& $8 m+3$ & none \\
$x^{8}+a x^{5}$ & $8 m+5$ & possibly $G F\left(13^{n}\right)$ for odd $n$ \\
& $8 m+7$ & possibly $G F\left(7^{n}\right)$ for odd $n$ \\
\hline
\end{tabular}

In conclusion we might ask whether, for each integer $k$, there exists a bound $N=N_{k}$ such that if $f(x)$ is of degree $2 k$ over $G F(q), f(x)$ will not permute $G F(q)$ if $q>N_{k}$.

Duke University

Durham, North Carolina

1. L. E. Dickson, "Analytic representation of substitutions, Ann. of Math., v. 11, 189697 , p. $65-120$.

\title{
Multistep Integration Formulas
}

\section{By A. C. R. Newbery}

A multistep formula for the approximate solution of an ordinary differential equation $x^{\prime}=f(x, t)$ has the form $\sum_{i=0}^{k} a_{i} x_{i}=h \sum_{i=0}^{k} b_{i} x_{i}{ }^{\prime}$. The formula is assumed to be stable, and to have optimum precision subject to this restriction; this means that a truncation error of the form $H h^{k+2} x^{(k+2)}(z)+0\left(h^{k+3}\right)$ is associated with the formula [1], where $x(t)$ is the exact solution of the differential equation and $H$ is a constant, which, like the $b_{i}$, depends on the choice of the constants $a_{i}$. A closed expression for the $b_{i}$ has already been given in [2, page 39], but it is considered worthwhile to tabulate the matrices which transform the $a_{i}$ into the $b_{i}$, to give an improved derivation of these matrices, and to extend the argument so that predictor coefficients can also be readily calculated.

The first task is, for a given $k$, to compute the elements $c_{i j}$ of a $(k+2) \times k$ 'corrector matrix' $C_{k}$, such that $b=C_{k} a$, where $b=\left\{b_{0}, b_{1}, \cdots b_{k}, H\right\}$ ' and $a=\left\{a_{1}, a_{2}, \cdots a_{k}\right\}^{\prime}$. (Note that $a_{0}$ is determined by the consistency condition $\sum_{0}{ }^{k} a_{i}=0$.) Using the notation of Antosiewicz and Gautschi [4, page 327] the relation between the required $b_{i}$ and the given $a_{i}$ is equivalent to the requirement that the linear functional $L x(t) \equiv \sum_{i=0}^{k}\left[a_{i} x(i)-b_{\imath} x^{\prime}(i)\right]$ should annihilate all

Received November 1, 1962. 
polynomials $x(t)$ of degree $\leqq k+1$. We define

$$
\begin{array}{ll}
\pi(t)=\prod_{i=0}^{k}(t-i), & \pi_{i}(t)=\pi(t) /(t-i), \\
\bar{\pi}(t)=\int_{0}^{t} \pi(u) d u, & \bar{\pi}_{i}(t)=\int_{0}^{t} \pi_{i}(u) d u .
\end{array}
$$

Then for $i, j \in\{0,1, \cdots k\}$ we have

$$
\bar{\pi}_{j}^{\prime}(i)=\pi_{j}(i)=0 \quad(i \neq j), \quad \bar{\pi}_{j}^{\prime}(j)=\pi^{\prime}(j),
$$

so that

$$
L \bar{\pi}_{j}(t)=\sum_{i=0}^{k} a_{i} \bar{\pi}_{j}(i)-b_{j} \pi^{\prime}(j) .
$$

Since $\bar{\pi}_{j}(t)$ is a polynomial of degree $k+1$, it must be annihilated by $L$; hence

$$
b_{j}=\frac{1}{\pi^{\prime}(j)} \sum_{i=1}^{k} a_{i} \bar{\pi}_{j}(i) \text {. }
$$

(Since $\bar{\pi}_{j}(0)=0$, the lower limit of summation may be taken as 1 ). In order to determine $H$, we set $x(t)=\bar{\pi}(t)$, so that $x^{\prime}(t)=\pi(t)$ and $x^{(k+2)}(t)=(k+1)$ !. When we form $L \bar{\pi}(x)$, we note that all the terms involving $b_{i}$ vanish; consequently

$$
H=\frac{1}{(k+1) !} L \bar{\pi}(t)=\frac{1}{(k+1) !} \sum_{i=1}^{k} a_{i} \bar{\pi}(i) .
$$

Combining the results (2), (3) into matrix form, we have for a given $k$,

$$
\begin{array}{r}
b=C a, \quad a=\left\{a_{1}, a_{2}, \cdots a_{k}\right\}^{\prime}, \quad b=\left\{b_{0}, b_{1}, \cdots b_{k}, H\right\}, \\
C=\left[c_{i j}\right], \quad c_{i j}=\bar{\pi}_{i}(j) / \pi^{\prime}(i) \quad(0 \leqq i \leqq k, 1 \leqq j \leqq k), \\
c_{k+1, j}=\bar{\pi}(j) /(k+1) !
\end{array}
$$

A 'predictor matrix' can be similarly derived; the vector $b$ is now defined by $b=$ $\left\{b_{0}, b_{1}, \cdots b_{k-1}, H\right\}, L$ is subject to the restriction $b_{k}=0$, and in (1) we define $\pi(t)=\prod_{i=0}^{k-1}(t-i)$. For a given $k$ the matrix $P$ is of dimension $(k+1) \times k$. The result is

$$
\begin{aligned}
b=P a, \quad p_{i j}=\bar{\pi}_{i}(j) / \pi^{\prime}(i) \quad(0 \leqq i \leqq k-1,1 \leqq j \leqq k), & \\
& p_{k j}=\bar{\pi}(j) / k !
\end{aligned}
$$

The matrix elements $c_{i j}, p_{i j}$ have been calculated exactly in rational arithmetic for $k=2 \cdots 8$, and the results are tabulated below. In order to avoid tabulating fractional elements, the lowest common denominator $D$ has been written above each matrix; thus for $k=2, c_{12}=\frac{32}{24}$. These matrices provide a compact tabulation of all the standard multistep formulas; if one ignores the last row of each corrector matrix, the last column gives the Newton-Cotes coefficients; the difference of the last two columns gives the Adams coefficients; various linear combinations 
of the columns give coefficients for the various radial and other formulas discussed in [3].

Predictor Matrices

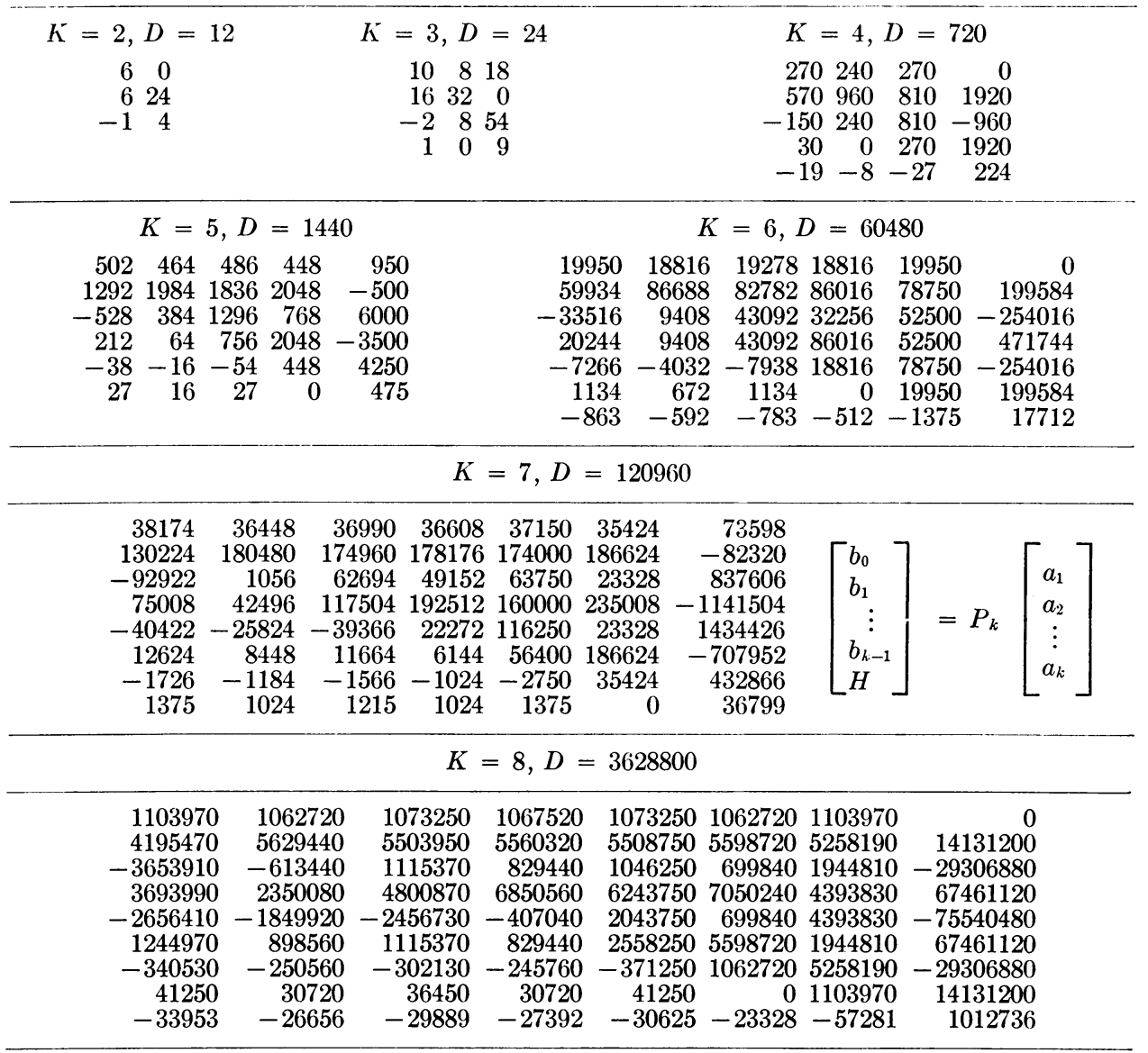

Corrector Matrices

\begin{tabular}{|c|c|c|}
\hline$K=2, D=24$ & $K=3, D=720$ & $K=4, D=1440$ \\
\hline 108 & $270 \quad 240 \quad 270$ & $\begin{array}{llll}502 & 464 & 486 & 448\end{array}$ \\
\hline 1632 & $570960 \quad 810$ & $\begin{array}{llll}1292 & 1984 \quad 1836 \quad 204\end{array}$ \\
\hline-28 & $-150240 \quad 810$ & $\begin{array}{lllll}-528 & 384 & 1296 & 76\end{array}$ \\
\hline 10 & $\begin{array}{rrr}30 & 0 & 270 \\
-19 & -8 & -27\end{array}$ & $\begin{array}{rrrr}212 & 64 & 756 & 2048 \\
-38 & -16 & -54 & 448 \\
27 & 16 & 27 & \end{array}$ \\
\hline
\end{tabular}

\begin{tabular}{rrrrrrrrrrrr}
\hline \multicolumn{3}{c}{$K=5, D=60480$} & \multicolumn{4}{c}{$K=6, D=120960$} \\
19950 & 18816 & 19278 & 18816 & 19950 & 38174 & 36448 & 36990 & 36608 & 37150 & 35424 \\
59934 & 86688 & 82782 & 86016 & 78750 & 130224 & 180480 & 174960 & 178176 & 174000 & 186624 \\
-33516 & 9408 & 43092 & 32256 & 52500 & -92922 & 1056 & 62694 & 49152 & 63750 & 23328 \\
20244 & 9408 & 43092 & 86016 & 52500 & 75008 & 42496 & 117504 & 192512 & 160000 & 235008 \\
-7266 & -4042 & -7938 & 18816 & 78750 & -40422 & -25824 & -39366 & 22272 & 116250 & 23328 \\
1134 & 672 & 1134 & 0 & 19950 & 12624 & 8448 & 11664 & 6144 & 56400 & 186624 \\
-863 & -592 & -783 & -512 & -1375 & -1726 & -1184 & -1566 & -1024 & -2750 & 35424 \\
& & & & & 1375 & 1024 & 1215 & 1024 & 1375 & 0
\end{tabular}




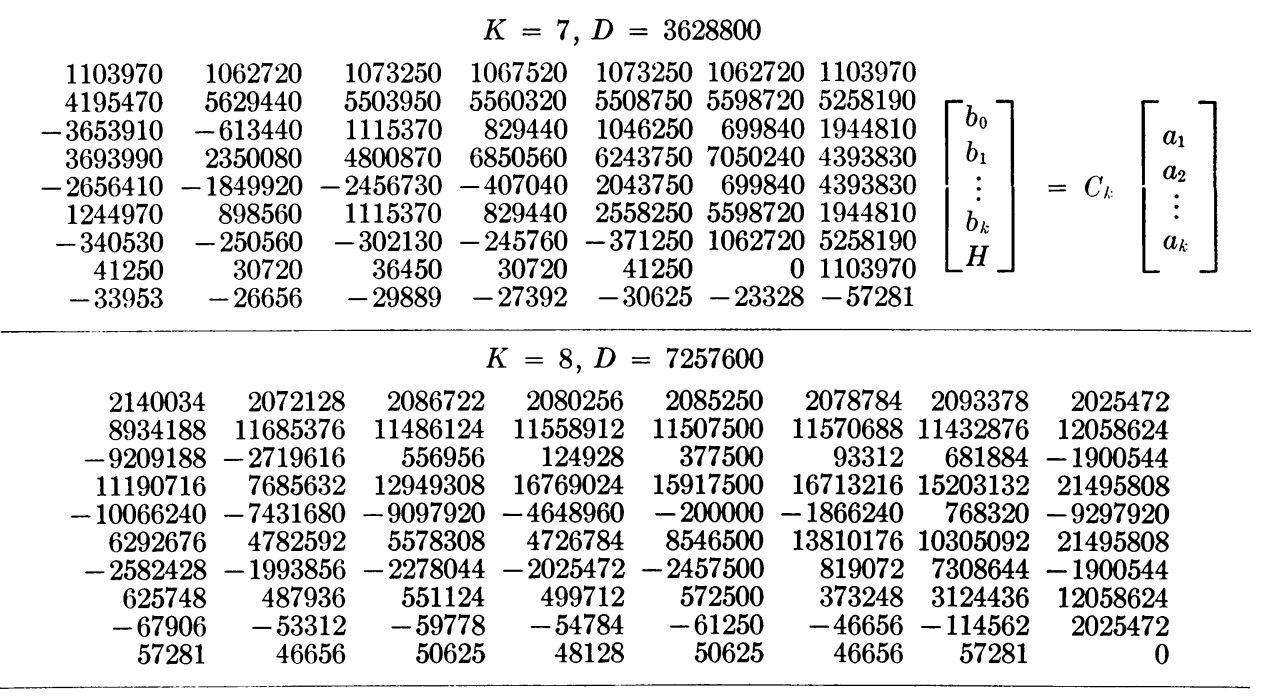

The Boeing Company

Renton, Washington

Now at

University of Alberta

Calgary, Alta, Canada

1. G. DAHLQUist, "Convergence and stability in the numerical integration of ordinary differential equations," Math. Scand., v. 4, 1956, p. 33-53.

2. T. E. Hull, \& A. C. R. NewBery, "Integration procedures which minimize propagated errors," J. Soc. Indust. Appl. Math., v. 9, 1961, p. 31-47.

3. - "Corrector formulas for multi-step integration methods," J. Soc. Indust. A ppl. Math., v. 10, 1962, p. 351-369.

4. H. A. Antosiewicz \& W. Gautschi, "Numerical methods in ordinary differential equations," A Survey of Numerical Analysis, Editor, J. Todd, McGraw-Hill Book Co., New York, 1962, p. 314-346.

\section{On the Non-Existence of Fibonacci Squares}

\section{By M. Wunderlich}

The Fibonacci sequence, $F_{n}$, is defined as follows:

$$
F_{1}=1 ; \quad F_{2}=1 ; \quad F_{n}=F_{n-2}+F_{n-1}
$$

for $n>2$.

A. P. Rollet [1] has posed the following problem. There are only three known Fibonacci numbers which are squares; $F_{1}=1, F_{2}=1$, and $F_{12}=144$. Are there any others? The purpose of this note is to announce that except for the known cases, $F_{n}$ cannot be a square for $n \leqq 1,000,000$, and to describe the computational method used to arrive at this result. The referee has kindly pointed out that the method used is somewhat analogous to familiar "exclusion" methods such as those described in [2].

Let $p$ be an arbitrary fixed prime number. With respect to this prime, denote by

Received November 20, 1962, revised April 10, 1963. 